\title{
Assessment of Pulmonary Valve and Pulmonary Stenosis
}

${ }^{1}$ A Subramanian, ${ }^{2} \mathrm{~V}$ Malik, ${ }^{3} \mathrm{R}$ Sreedhar

\section{ABSTRACT}

The Fellow's Corner is a short treatise on material relevant for the learning of trainees in cardiac anesthesia. The first in this series is on the TEE assessment of the pulmonary valve.

Keywords: Assessment, Transesophageal, Pulmonary valve.

How to cite this article: Subramanian A, Malik V, Sreedhar R. Assessment of Pulmonary Valve and Pulmonary Stenosis. J Perioper Echocardiogr 2014;2(1):40-41

Source of support: Nil

\section{Conflict of interest: None}

\section{ANATOMIC CONSIDERATIONS}

The pulmonary valvular apparatus consists of three leaflets (anterior, left and right), their associated sinuses of valsalva and a sinotubular junction. The pulmonary valve has an area of approximately $2 \mathrm{~cm}^{2} / \mathrm{m}^{2}$ body surface area. It has nodules of Arantii (small fibrous nodules in the free margin of the cusp) and lunulae (thin half-moon shaped areas along the free edge of each cusp). The pulmonary valve is an anterior structure. Compared to the aortic valve, it is at a greater distance from the ultrasound transducer located in the esophagus. The valve least well imaged using TEE is the pulmonary valve. ${ }^{1}$

\section{TEE VIEWS FOR THE PULMONARY VALVE ${ }^{2}$}

1. Midesophageal RV inflow-outflow view

2. Midesophageal aortic valve short axis view

3. Transgastric views

Midesophageal right ventricular inflow-outflow view is a popular scan plane for imaging the pulmonary valve using TEE. Here, the pulmonary valve is seen in its long axis and the aortic valve in short axis. In the midesophageal aortic valve short axis view, the pulmonary valve is again seen in long axis (Fig. 1). However, it is usually not possible to

\footnotetext{
${ }^{1}$ Senior Resident, ${ }^{2}$ Additional Professor, ${ }^{3}$ Professor

1,2Department of Cardiac Anesthesiology, All India Institute of Medical Sciences, New Delhi, India

${ }^{3}$ Department of Anesthesia, Division of Cardiac Anesthesia Sree Chitra Tirunal Institute for Medical Sciences and Technology, Thiruvananthapuram, Kerala, India

Corresponding Author: $V$ Malik, Additional Professor Department of Cardiac Anesthesiology, All India Institute of Medical Sciences, New Delhi, India, e-mail: vishwasmalik@ hotmail.com
}

reliably interrogate the PV by Doppler methods in these mid-esophageal views owing to suboptimal beam alignment

For Doppler interrogation of the PV transgastric right ventricular inflow-outflow view is useful. This view can allow accurate alignment of the Doppler beam with the area of subvalvar/valvular stenosis through the RV outflow tract (transducer position (120 to $145^{\circ}$ ) can be used (Fig. 2). Other windows in which the pulmonary outflow tract may be interrogated include the deep transgastric view in which both ventricular outflow tracts can be profiled in a single window. Finally, in the upper esophageal aortic arch short axis view, the distal right ventricular outflow tract, pulmonary artery, and pulmonary valve can be seen beneath the aortic arch. In this view, the pulmonary valve flow is well aligned with the ultrasound beam vector and this is thus, a good view for quantifying pulmonary stenosis (Fig. 3).

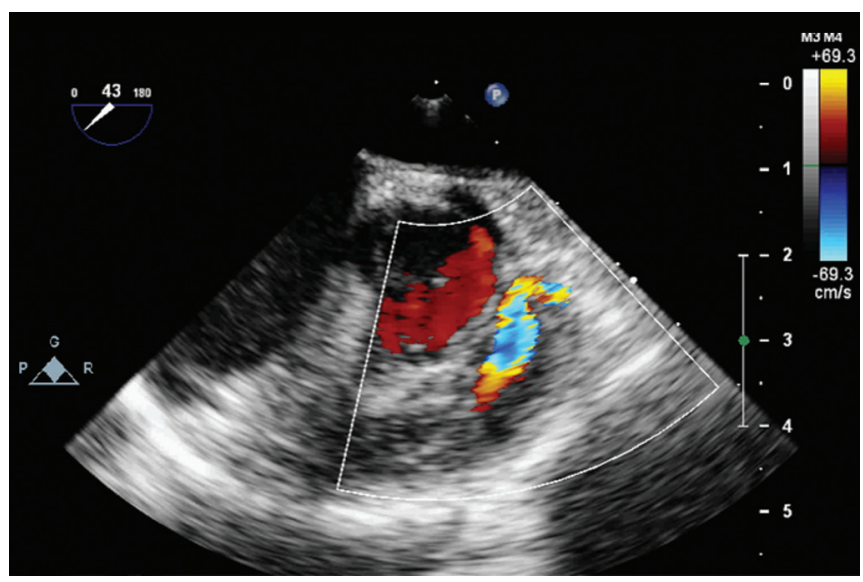

Fig. 1: RV inflow-outflow view

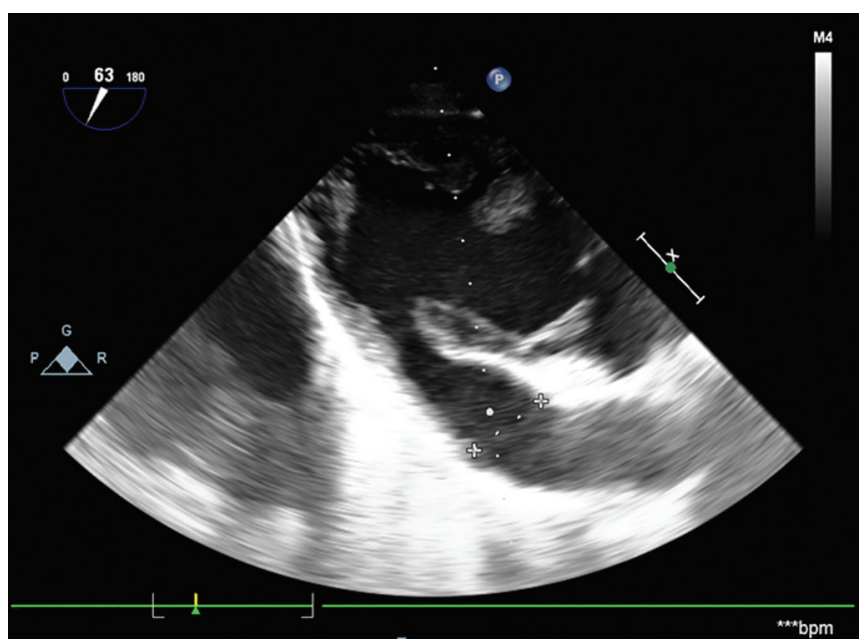

Fig. 2: Transgastric RV inflow-outflow view 


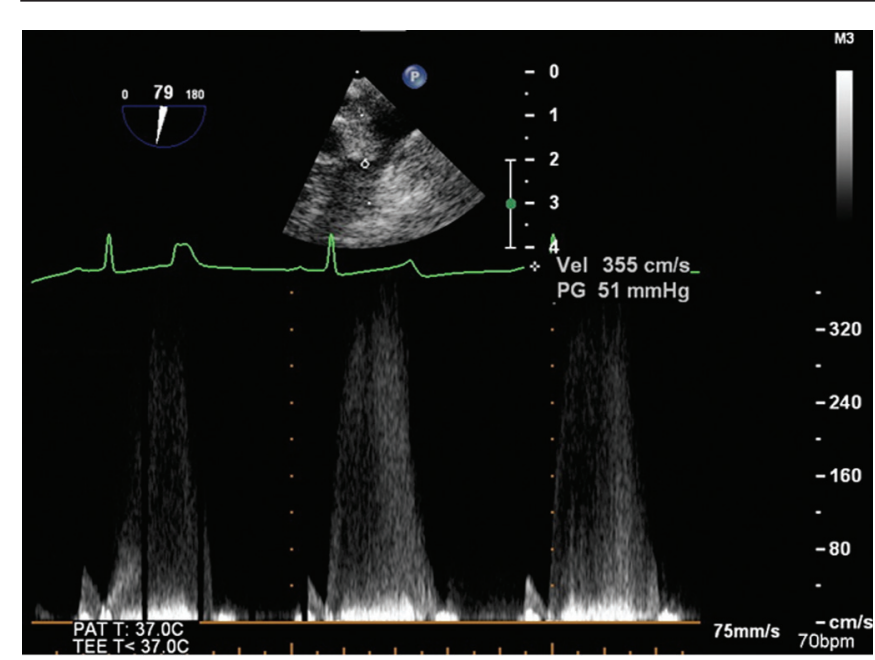

Fig. 3: Upper esophageal aortic arch short-axis view (with CW across RVOT)

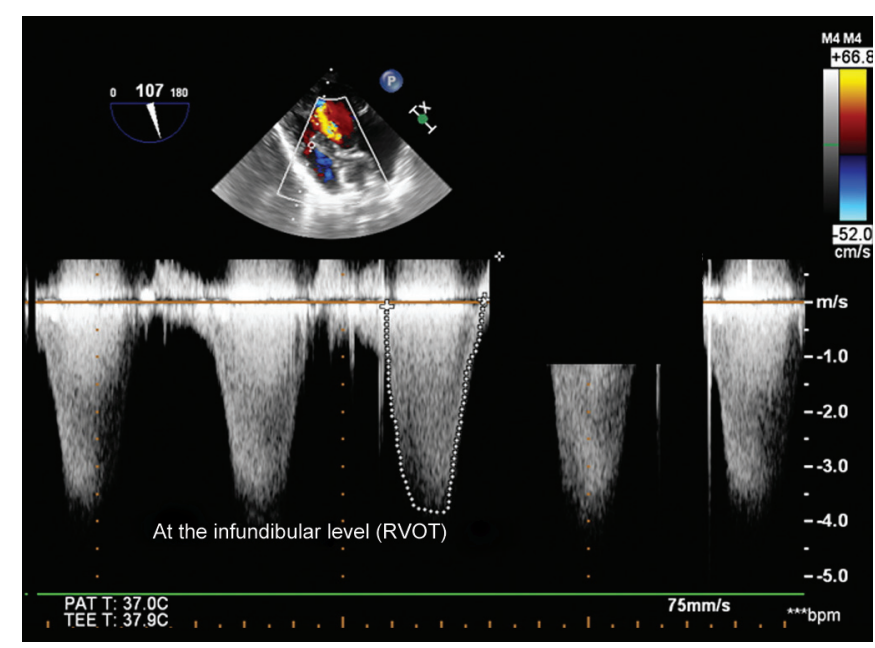

Fig. 4: Gradient at the infundibular level. Note the characteristic mid-late systolic rise in the peak velocity (transgastric PV view)

Such quantification requires estimation of the peak or mean transvalvular pressure gradient with continuous wave Doppler. PS is considered mild when the peak gradient across the $\mathrm{PV}$ is less than $30 \mathrm{~mm} \mathrm{Hg}$, moderate when the gradient is 30 to $50 \mathrm{~mm} \mathrm{Hg}$, and severe when the gradient is more than $50 \mathrm{~mm} \mathrm{Hg}$. Mean Doppler and catheter-based gradients correlate better than peak gradients.

Muscular infundibular obstruction is frequently characterized by a late peaking systolic jet that appears 'dagger

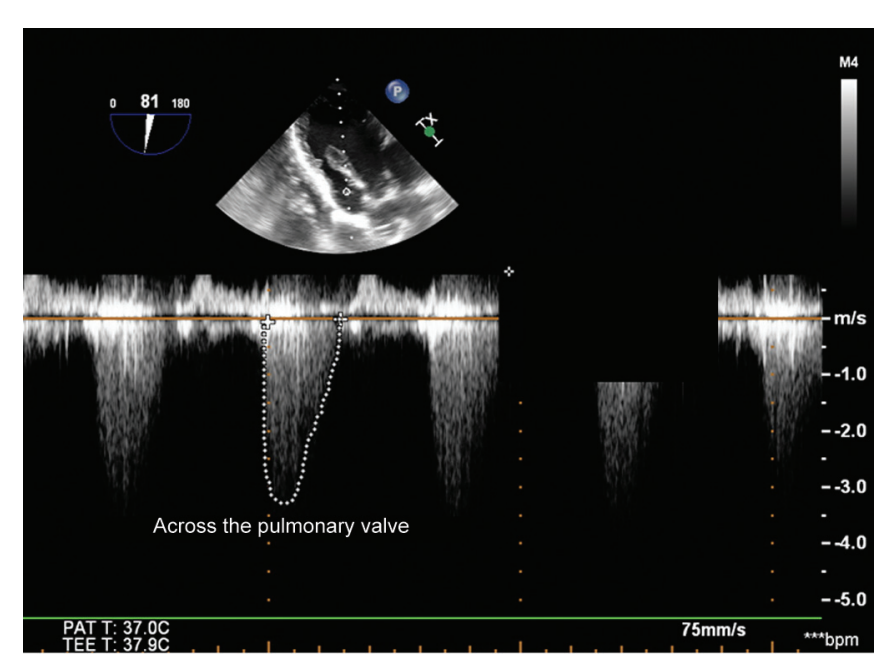

Fig. 5: Gradient across the pulmonary valve. Note the characteristic early systolic rise in the peak velocity (transgastric PV view)

shaped ${ }^{3}$, reflecting the dynamic nature of the obstruction; this pattern can be useful is separating dynamic muscular obstruction from fixed valvular obstruction, where the peak velocity is generated early in systole (Figs 4 and 5). These can be further anatomically defined using pulse wave Doppler.

Pulmonary stenosis is usually congenital in origin. It may be subvalvular, valvular, or supravalvular. Post stenotic pulmonary artery dilatation may be present. Carcinoid heart disease is one of the few causes of pulmonary stenosis in the adult.

\section{REFERENCES}

1. Kumbharathi RB, Taneja R, Mehra R, Quantz MA, Guo LR, Bainbridge DT. Assessment of tricuspid and pulmonary valves using epicardial and transesophageal echocardiography: a comparative study. J Cardiothorac Vasc Anesth 2012;26: 32-38.

2. Barbeito A, Schroeder RA, Bar- Yousef S, Mark JB. Assessment of tricuspid and pulmonary valves. In Savage RM, Aronson S, Shernan SK, editors. Comprehensive Textbook of Perioperative Transesophageal Echocardiography. Baltimore Wolters Kluwer Lippincott Williams Wilkins 2010;236-251.

3. Shah BN, Curzen NP. Reversible systolic anterior motion of the mitral valve and left ventricular outflow tract obstruction in association with Takotsubo syndrome. Echocardiography 2011; 28:921-924.

Note: Figures 1 and 3 have been obtained from PGI Chandigarh Echo archives. 